\title{
La petición de pruebas complementarias en somatizadores en Atención Primaria: Un estudio cualitativo
}

\author{
J. García-Campayo* \\ E. Dueñas** \\ M. Alda*** \\ L.M. Claraco** \\ E. Arévalo**
}

* Psiquiatra y Profesor Asociado, Unidad de Trastornos Somatomorfos, Hospital Universitario Miguel Servet y Universidad de Zaragoza

** Médico de Familia, Hospital Universitario Miguel Servet y Universidad de Zaragoza

*** MIR Psiquiatría, Hospital Universitario Miguel Servet y Universidad de Zaragoza

ESPAÑA

RESUMEN - Objetivo. Determinar la razón para la petición de pruebas complementarias en pacientes somatizadores de atención primaria.

Métodología. Estudio cualitativo basado en entrevistas en profundidad individuales y en grupos focales. Se estudió a treinta y siete somatizadores pertenecientes a diez consultas de atención primaria de Zaragoza, España.

Resultados. Fueron identificados cuatro subgrupos de pacientes en dependencia de sus características personales, experiencias previas negativas con médicos, confianza en la tecnología o papel activo en las cuestiones sobre la salud. Estos grupos eran: 1. Pacientes que tienden a confiar en la explicación somática de los síntomas, 2. Pacientes marcados por experiencias médicas negativas, 3 . Buscadores de seguridad y 4 . Buscadores de tecnología. Además existe un pequeño grupo de pacientes que no encajan con ninguno de estos modelos.

Conclusiones. Hay varios modelos que explican el comportamiento de un alto porcentaje de pacientes. En un individuo en particular, este modelo parece estable en el tiempo y predecible, por lo que se podría diseñar un tratamiento específico. Sin embargo, un cierto porcentaje de somatizadores no se ajusta a ningún modelo, su comportamiento parece cambiar a lo largo del tiempo y no es fácil desarrollar un tipo de tratamiento específico. 


\section{Introducción}

La somatización ha sido definida como "la experiencia y comunicación de malestar psicológico en forma de síntomas físicos" (Lipowski 1988). Este es un fenómeno común en la población general (Escobar et al. 1989) y representa el $25-30 \%$ del total de las consultas de atención primaria (Lobo et al. 1996). Añadido a la alta prevalencia, la somatización es importante para el sistema médico porque es responsable de al menos el $10 \%$ del coste total de salud en los países desarrollados (Barsky et al. 1986). Aunque la causa de la somatización no está clara, la mayoría de los autores defienden un modelo etiológico multicausal interactivo (Mayou et al. 1995), incluyendo aspectos tales como mecanismos fisiopatológicos, afrontamiento, procesos familiares o ganancias secundarias.

En estos últimos años, la somatización aguda ha sido objeto importante de investigación porque se ha sugerido que las formas de somatización crónica vistas en las consultas de psiquiatría provienen de la evolución de las somatizaciones agudas y subagudas encontradas en atención primaria (Craig et al. 1993). Se esperaba que la comparación de las características clínicas y de personalidad que presentan los somatizadores y los psicologizadores (estos últimos son pacientes que expresan su malestar psicológico en forma de síntomas psicológicos) daría una pista sobre la etiología de la somatización, pero los resultados han sido poco concluyentes (García-Campayo et al. 1999).

La mayoría de las investigaciones realizadas hasta ahora en somatización han utilizado métodos cuantitativos. Sin embargo, los métodos cualitativos están siendo progresivamente más utilizados en las ciencias de la salud poque complementan a los cuantitativos. Algunas técnicas tales como la observa- ción, las entrevistas en profundidad y los grupos focales se pueden utilizar para proporcionar una descripción y comprensión de la situación o comportamiento, siendo un hecho preliminar esencial para los estudios cuantitativos (Mays et al. 1996). De hecho, en el campo de los trastornos somatomorfos, los métodos cuantitativos se han usado para mejorar la comprensión del proceso de consulta en pacientes médicos en general (Cromarty 1996) y de los trastornos somatomorfos en particular (Salmon et al. 1999), o el problema de los frecuentadores (Neal et al. 2000).

El objetivo de este estudio es analizar, desde un punto de vista cualitativo, algunos aspectos del proceso psicológico de la consulta médica en pacientes que presentan somatización con especial énfasis en la razón para solicitar pruebas complementarias o la derivación a otros especialistas (si ha existido), y las espectativas de los pacientes sobre los médicos.

\section{Metodología}

\section{Selección de pacientes y reclutamiento}

Cuarenta pacientes de atención primaria atendidos por diez médicos de familia de los centros de salud de Torrero y San Marcial, en Zaragoza, España, utilizando el criterio de Goldberg para la presentación de somatizadores (Bridges et al. 1985), usando el SPPI (Lobo et al. 1993), una entrevista psiquiátrica especficamente diseñada para pacientes de atención primaria y utilizada extensamente en somatizadores (Lobo et al. 1996, García-Campayo et al. 1996 y 1997), fueron invitados a participar en el estudio. De ellos, 3 pacientes rechazaron y los restantes firmaron el consentimiento informa- 
do y fueron incluidos. Las muestras representativas son cruciales en los estudios cuantitativos pero no en los estudios cualitativos (Mays, Pope 1995), en los cuales la cuestión clave es la obtención de una extensa representación de la población estudiada. Por esta razón, aseguramos una variedad suficiente en relación a la edad, sexo, nivel cultural o profesión. La recogida de datos fue llevada a cabo durante el periodo de mayo de 1999 a abril del 2000.

\section{Valoración del paciente}

Se emplearon los siguientes métodos cualitatitivos:

\section{Entrevista en profundidad}

Utilizamos entrevistas semiestructuradas en las que algunos puntos fueron abarcados con gran detalle y las cuestiones subsiguientes estaban basadas en lo que el paciente decía (Mays et al. 1996). Las entrevistas duraban una media entre 60 y 90 minutos. Eran grabadas y, después, transcritas por un investigador. Las cuestiones estaban siempre referidas a las dos o tres últimas consultas médicas del paciente. Las preguntas abiertas en la entrevista eran. “¿Cuándo considera que un médico ha estudiado suficientemente su caso? Otras cuestiones incluidas sistemáticamente eran: “¿Solicita ocasionalmente pruebas complementarias y por qué?”, “¿Cómo sabe que una prueba complementaria es necesaria?" Los pacientes eran alentados a dar respuestas exhaustivas de sus ideas y experiencias. Sus respuestas fueron facilitadas para explicar y ampliar sus descripciones espontáneas. No se hicieron preguntas sobre ideas o creencias no mencionadas espontáneamente por los pacientes.

\section{Grupos focales}

Todos los pacientes que eran entrevistados previamente, eran invitados a participar en los grupos focales. Treinta de estos pacientes aceptaron asistir a alguno de ellos. Estos grupos estaban compuestos por 4-6 pacientes para aprovechar la comunicación entre los participantes y generar datos. Se realizaron cinco grupos focales y los participantes eran selecionados en base a las máximas diferencias en las características sociodemográficas y los resultados de la entrevista exhaustiva. Este método es particularmente útil para explorar los conocimientos y experiencias y se puede usar para examinar no sólo lo que la gente piensa, sino cómo y por qué piensan de esa forma (Mays et al. 1996). Las sesiones duraban 90-120 minutos y la interacción entre los participantes era estimulada. Un observador no participante tomaba notas en la sesión y las conclusiones principales eran apuntadas en una lista para ser discutidas por el grupo.

\section{Análisis de lo encontrado en las entrevistas}

\section{Entrevista en profundidad}

La transcripción de los temas era analizada usando una forma temática de analizar el contenido (Weber et al. 1990) siguiendo los tres pasos descritos por estudios previos de investigación: El primer paso es identificar temas comunes en las respuestas de los pacientes y clasificarlos de acuerdo a su significado. El número de códigos producido de este modo en respuesta a cada pregunta revela la complejidad de los pensamientos del paciente y enfatiza el carácter único de cada discurso. Sin embargo, varios temas dominantes eran evidentes, los cuales eran confirmados por los datos de la mayoría de las entrevistas (Riley 1990, Mays et al. 1995). El segundo paso era agrupar esos temas que compartían un significado común en categorías. El tercer paso era contar el número de pacientes que había expresado cada tema en 
su informe para indicar su frecuencia relativa en la muestra. Un cuarto paso adicional era construir, cuando fuese posible, un esquema etiológico basado en las interrelaciones de los datos. Finalmente, nos concentramos en los casos desviados, es decir, en los pacientes que parecían contradecir las explicaciones más importantes. Para minimizar el sesgo en el análisis, un segundo investigador independientemente repetía el proceso y si aparecía alguna discrepancia se resolvía por consenso.

\section{Grupos focales}

El análisis de los datos del grupo focal era similar a los otros datos cualitativos (Mays, Pope 1995, 1996) para comparar la discusión de temas similares y examinar como estos se relacionan a variables dentro de la población muestral. Es muy importante distinguir entre opiniones individuales y consenso de grupo y dar importancia a los casos desviados (Mays, Pope 1996). De nuevo, dos investigadores distintos analizaron independientemente los datos y las diferencias fueron resueltas por acuerdo.

\section{Resultados}

\section{Características de la muestra}

La muestra final de pacientes $(\mathrm{N}=37)$ tenía una edad media de 43 años (rango 2163 años). El 75\% eran mujeres (28 de 37), el $94,5 \%$ (35 de 37) había recibido al menos educación elementaria y el 54\% (20 de 37) trabajaban. Todas las clases sociales estaban representadas con un predominio de la clase media-baja como es usual en Sistema Nacional de Salud español.

\section{Respuestas a preguntas específicas}

Las respuesta a una de las cuestiones específicas más importantes: “¿Por qué solicita más pruebas complementarias o derivaciones?" es resumido en la tabla I describiendo temas, categorías y número de pacientes de cada uno de ellos. El mismo proceso se ha seguido con las otras cuestiones más importantes.

Tabla I

Respuestas a la pregunta: ¿Por qué solicita más pruebas complementarias? Temas y categorías

Temas Categorías

Nunca se le ocurre al paciente pedir pruebas

Confianza básica en los médicos

Siempre negocia con el médico cualquier duda o pregunta

Error de un médico con el paciente $\quad$ Experiencias previas con los médicos

Error de un médico con la familia/amigos negativas

Conocimiento de errores médicos por otras personas

Descripción de errores médicos en medios de comunicación

Muerte repentina de familiar/conocido

Énfasis de los medios de comunicación

Miedo a la muerte o a la enfermedad

en la prevención/diagnóstico precoz

Miedo a la muerte

Miedo a enfermedades particulares: SIDA, cáncer.

Seguridad de su mala salud

Conocimiento de nuevas pruebas diagnósticas

Sentimiento de más seguridad con las pruebas de alta tecnología

Desacuerdo del médico con las recomendaciones de Internet

Desacuerdo del médico con las recomendaciones de los libros/ medios de comunicación
Confianza en la tecnología y los medios de comunicación 


\section{Modelos de comportamiento de los diferentes grupos de pacientes}

Hay varias características de los pacientes que se ajustan a los diferentes patrones de comportamiento en muchos de los pacientes. Sin embargo, no todos los pacientes muestran estas asociaciones y hay un número de casos "desviados" difíciles de explicar y agrupar. Nosotros hemos encontrado los siguientes grupos de pacientes bien definidos de acuerdo a la razón para solicitar pruebas complementarias:

1. Pacientes que creen en el médico que tienden a fiarse de las explicaciones somáticas de sus síntomas (11 pacientes): Este parece ser el grupo más numerosos. Podían o no haber tenido experiencias médicas previas pero en ningún caso habían sido experiencias negativas. Como consecuencia, mantenían una confianza básica en los médicos y aceptaban plenamente pruebas auxiliares usadas por el médico para completar su criterio. Ejemplos de transcripciones que apoyan estos datos son las siguientes:

Paciente n. ${ }^{\circ}$ 2: "Mi familia y yo no hemos consultado a los médicos demasiado pero, en general, estamos satisfechos con ellos."

Investigador: “¿Piensa que los médicos son fiables o no?"

P: "Por supuesto. Obviamente, no todos son iguales. La televisión está llena de casos de gente que demanda a los médicos por errores. Yo pienso que errar es humano y puede pasar ocasionalmente. Mi experiencia es buena y no tengo ninguna razón para no confiar en los médicos."

I: "¿Qué hay sobre exploraciones médicas como los Rayos X, TAC, ECG etcétera? ¿Deberían los médicos pedirlas sistemáticamente en cada consulta o no?"
P: "No pienso que estas pruebas sean necesarias en cada consulta. Muchas veces el médico puede obtener un diagnóstico con un simple análisis de sangre. Ellos saben cuando es necesario solicitar más pruebas."

I: “¿Pero se siente mejor si se realiza una radiografía o no?"

P: "No necesariamente. Si el médico no la pide está bien para mí."

Para estos pacientes, el final de las pruebas complementarias lo decide el médico cuando ha alcanzado un diagnóstico.

I: “¿Cuándo considera que un médico ha estudiado suficientemente su caso?"

P: "Bueno, cuando el médico considera que es suficiente y me dice el diagnóstico y el tratamiento a seguir."

I: “Así que el diagnóstico y el tratamiento de la enfermedad es el final del estudio para usted."

P: "Si, eso pienso."

I: ¿Le ha pasado alguna vez que el médico ha acabado el diagnóstico de su enfermedad pero usted ha seguido pidiendo más pruebas?"

P: "No lo recuerdo. Normalmente mi médico pide pruebas hasta que dan un diagnóstico y eso es suficiente. En alguna ocasión yo he podido mencionar una prueba que hemos escuchado en la tele o así, pero si mi médico me explica que la prueba no es necesaria por esto y por eso, yo lo acepto sin ningún problema."

El problema de esta clase de somatizadores no es el médico sino el diagnóstico. No están preparados para aceptar que los síntomas físicos son a menudo ocasionados por malestar psicológico. La conmoción que produce esta información hace que el paciente discuta con el médico y solicite 
nuevas pruebas o exámenes. Sin embargo, debido a la confianza en sus médicos, aceptan un tratamiento psicofarmacológico de prueba y, si el tratamiento funciona, como suele pasar normarmente, aceptan las explicaciones psicológicas de la enfermedad.

I: “Qué pensó cuando su médico le explicó que su vértigo podía estar causado por una causa psicológica? ¿Lo aceptó facilmente?

Paciente n. ${ }^{\circ}$ 6: “Al principio, no lo entendí. Siempre he pensado que los síntomas físicos son debidos a causas físicas. Era la primera vez que yo había escuchado algo sobre eso. Así que yo le pedí al médico nuevas pruebas porque pensaba que en algo se podía haber equivocado. El médico me dio algunas pastillas y acordamos que si los tranquilizantes no eran efectivos en una semana, él revisaría el diagnóstico y pediría nuevas pruebas. Para mi sorpresa, mejoré mucho con las pastillas y acepté la causa psicológica del problema."

2. Pacientes marcados por experiencias médicas previas negativas (8 pacientes): Estos parecen ser un grupo diferente de somatizadores marcados por una experiencia médica negativa, real o percibida, que ellos o un familiar o amigo ha tenido.

"Mi madre falleció porque su médico fue incapaz de detectar un cáncer de mama hasta que fue demasiado tarde. Ella sufrió fatiga, dolor en el pecho, etcétera. El médico le pidió varios análisis de sangre y una RX de tórax que tardó mucho tiempo en pedirla. Cuando mi madre fue remitida al oncólogo, el cáncer estaba tan extendido que era inoperable. Desde entonces ya no creo en los médicos" (paciente n. ${ }^{\circ} 8$ ).

En general, basándonos exclusivamente en las declaraciones de los pacientes, es imposible saber si hubo realmente un error médico o fue solamente una percepción subjetiva por parte del paciente. Cuando el entrevistador pregunta sobre cómo sabe que ocurrió un error, estos pacientes se sientes incómodos y tienden a contestar que porque lo saben. Sin embargo, ninguno de los pacientes de nuestra muestra había denunciado a un médico por mala práctica. Cuando se les preguntó por este hecho, la mayoría de los somatizadores contestaron que habría sido inútil por el corporativismo de la profesión. En cualquier caso, como éste y otros pacientes claramente explican, la experiencia negativa ha producido una desconfianza básica en los médicos.

I: "¿Qué hizo cuando se dio cuenta de que había habido un retraso en el diagnóstico de la enfermedad de su madre?"

Paciente n. ${ }^{\circ}$ 8: "Estaba muy enfadada con el médico y le dije que no era un profesional porque mi madre se podría haber recuperado si el hubiese actuado correctamente."

I: “¿Cuál fue la respuesta del médico?”

P: "Ya sabe,..., él echó la culpa a la enfermedad diciendo que todo lo que se podía haber hecho se había hecho. Pero no admitió su error."

\section{I: “Pensó en denunciarlo?”}

P: "Sí, por supuesto,...pero ya sabe...porque usted también es médico, que ningún médico habla mal de otro colega, así que la denuncia habría sido inútil. El corporativismo médico es alto"

I:”¿Le ha afectado esta experiencia de alguna manera en su relación o confianza en los médicos?"

P: "Por supuesto,...sé que muchos médicos son buenos pero además son humanos y los errores no son sólo posibles sino que son además comunes. El paciente tiene que estar alerta para evitar los errores. Desde entonces, yo estoy bastante suspicaz sobre este hecho, y verifico todas las recomendaciones de mi médico." 
La confianza de estos pacientes ha sido desplazada del médico a las máquinas de alta tecnología como el TAC, el PET, el SPECT y similares.

"Hoy, la tecnología médica ha avanzado mucho y contar exclusivamente con la opinión médica es poco científico. Estar seguro de la buena salud de uno, implica la total normalidad de las pruebas complementarias y exámenes. Estas pruebas están hechas por máquinas y no pueden equivocarse, pero los médicos, que son humanos, pueden hacerlo" (paciente n. $^{\circ} 15$ ).

Finalmente, la explicación psicológica de los síntomas médicos es considerada por este grupo como un riesgo porque esta explicación puede disfrazar una falta de conocimientos sobre la enfermedad. Por esta razón, una explicación psicológica es sólo aceptable cuando todas las pruebas son normales, pero no sólo como consecuencia de la entrevista médica.

"Ciertamente, el malestar psicológico puede producir dolor en algunas ocasiones, pero yo me temo que esta es una simple explicación muy fácil para el médico. Ellos dan este diagnóstico y dejan pasar el tiempo. Si es verdad, los síntomas pueden desaparecer. La cuestión es cuando ellos cometen un error y la causa era una grave enfermedad: Muchas veces habría sido tarde y el tratamiento sería más difícil. Yo no creo que algo es "causado por los nervios" sólo porque el médico lo dice así. Yo necesito la confirmación de unos RX o de un SPECT" (paciente n. ${ }^{\circ}$ 7).

3. Buscadores de seguridad (6 pacientes): Hay pacientes que presentan rasgos de personalidad hipocondríaca a pesar de no haber experimentado acontecimientos médicos negativos. Confían tanto en los médicos como en los aparatos pero necesitan con frecuencia seguridad.
Paciente n. ${ }^{\circ}$ 5: "Siempre he sido una persona que estaba preocupada por el funcionamiento de mi cuerpo. Me gusta tomarme el pulso ocasionalmente y la tension arterial regularmente. Tengo cuidado con mi dieta y trato de no comer demasiada grasa o azúcar. Además, me impresiono facilmente con la muerte o la enfermedad de un amigo y durante varios días, pienso que me puede pasar a mí y me observo con más frecuencia".

I: “¿Cuál es la razón para este comportamiento?"

P: "No lo sé. Siempre he sido así."

I: "¿Usted o alguien que conozca ha tenido experiencias negativas con los médicos?"

P: "No,...no recuerdo nada digno de mención."

I: “¿Podemos decir que en general confía en los médicos?"

P: "Por supuesto, puede decirlo. No tengo nada contra los médicos. El problema es que suelo pensar en los síntomas que tengo una y otra vez. Entonces, muchas dudas o preguntas surgen así que voy al médico para preguntar por estos síntomas. Cuando el médico me contesta y me dice que todo está bien me tranquilizo, pero si los síntomas permanecen el ciclo se repite una y otra vez."

I: "Confia menos en los médicos que en las pruebas complementarias o los reconocimientos?"

P: "No, por igual. Los médicos piden las pruebas y las interpretan así que es lo mismo."

Las explicaciones psicológicas de los síntomas somáticos son aceptadas por un tiempo, pero requiere reconfirmación de vez en cuando.

I: “¿Puede aceptar una explicación psicológica para sus síntomas físicos?” 
Paciente n. ${ }^{\circ}$ 14: "Sí, yo creo en mi médico y puedo aceptar su explicación y me siento relajado durante algún tiempo. El problema es después de algún tiempo, si los síntomas continúan, temo que una peligrosa enfermedad puede haberse desarrollado después de la visita y necesito ir al médico de nuevo para hacer más pruebas."

4. Buscadores de tecnología (3 pacientes): Estos pacientes comparten ciertas características con el segundo grupo pero ellos no han tenido experiencias negativas con la medicina. Ponen más confianza en los aparatos que en los médicos, pero no tienen desconfianza médica. Están convencidos de que la alta tecnología es importante pero no como resultado de los errores médicos, sino debido a una profunda confianza en la tecnología. Generalmente, son jóvenes, con un nivel educativo alto y un buen conocimiento de medicina. Están muy interesados en leer literatura médica y en la obtención de información a través de varias fuentes, principalmente internet. Esta parece ser la forma de "probar" los conocimientos y habilidades del médico. Además son activos en los temas de salud y quieren participar en su propio tratamiento.

"Previo a cualquier consulta, me gusta haber leído algo sobre mi posible diagnóstico y pruebas a realizar. Frecuentemente uso internet para estar informado" (paciente 1).

Las pruebas complementarias se solicitan cuando hay discrepancias entre el médico y la información obtenida en internet. Las explicaciones psicológicas son aceptadas pero tienen que estar apoyadas por investigaciones médicas.

I: "Cuando pide más pruebas complemementaria o una derivación de su médico, ¿cuál es la razón?”

Paciente n. ${ }^{\circ}$ 1: “Cuando voy al médico normalmente he buscado en internet infor- mación sobre los síntomas. Espero que la información del médico sea coherente con lo que he leído."

I: “Así que si el médico le da una información o un diagnóstico diferente del que ha leido pide una derivación?"

P: "Bueno, no de esa forma. Discuto todas mis dudas con el médico. Generalmente, yo estoy satisfecho con sus respuestas. Sólo ocasionalmente no estoy contento con las explicaciones del médico y pido pruebas que yo considero necesarias para el diagnóstico o pido una derivación a un especialista."

I: “¿Puede aceptar una explicación psicológica de sus síntomas físicos?"

P: "Por supuesto. El problema no es que la explicación sea física o psicológica, sino el estar apoyada por investigaciones médicas."

5. Casos desviados (9 pacientes): Estos pacientes no pueden ser incluidos en ninguno de los grupos anteriores porque muestran características diferentes, algunas de ellas pertenecientes a algunos de los grupos, pero sin conformar un patrón bien definido. En este grupo todos los pacientes muestran una serie de características específicas que los diferencia de todos los demás individuales y requieren su propio modelo etiológico. Además, estas características pueden variar a lo largo del tiempo. Las explicaciones aplicadas a los otros cuatro grupos de pacientes son inútiles para estos pacientes. La importancia de este grupo es tener claro que algunos somatizadores presentan patrones indefinidos, cambiantes a lo largo del tiempo, dificilmente explicables basándose en experiencias previas o personalidad $\mathrm{y}$, como consecuencia, hacen mucho más difícil su manejo. 


\section{Discusión}

Este es, en nuestro conocimiento, el primer estudio cualitativo en la petición de pruebas complementarias en atención primaria en somatizadores agudos. Hay varios resultados clave provenientes de este estudio:

1. El proceso por el que los somatizadores toman decisiones individuales para la petición de pruebas complementarias o de derivaciones es compleja, y parece fuertemente influenciada por las características de personalidad y las experiencias pasadas con los médicos y las enfermedades. Los temas más importantes son los siguientes: Ciertos rasgos de personalidad (atribución de síntomas somáticos e hipocondriasis), experiencias previas negativas con los médicos (reales o percibidas), confianza en la tecnología o papel activo en los problemas de salud.

2. Hay varios patrones que explican el comportamiento de muchos pacientes: En un paciente en particular este patrón parece estable en el tiempo y predecible, así que un tratamiento específico aproximado se puede diseñar para cada uno de estos patrones. Los cuatro patrones detectados fueron los siguientes:

- Pacientes que tienden a fiarse de la explicación somática de sus síntomas: Individuos que, probablemente como consecuencia de una alta puntuación en el rasgo de personalidad llamado atribución somática de los síntomas (Robbins, Kirmayer 1991), consideran cualquier explicación psicológica de la enfermedad como inaceptable y necesitan confirmación con las pruebas complementarias.

- Pacientes marcados por experiencias médicas previas negativas: Pacientes que, debido a experiencias previas negativas con la medicina reales o percibidas, han perdido su confianza en los médicos y sólo creen en la tecnología.
- Buscadores de seguridad: Pacientes que confían en los médicos, con un alto nivel de hipocondriasis, que necesitan periódicamente reasegurarse en la ausencia de cualquier enfermedad.

- Buscadores de tecnología: Gente instruida que son activos en sus problemas de salud, y están bien informados sobre las patología médicas. Estas personas esperan que el médico confirme su propia hipótesis previa sobre su enfermedad.

3. Un cierto porcentaje de somatizadores no se ajustan a ningún patrón: El origen de su comportamiento, que parece cambiar a lo largo del tiempo, no es fácil de explicar y tratamientos específicos para ellos son difíciles de desarrollar.

4. Este tema no es un tabú para los pacientes, y ellos están preparados para discutirlo a pesar de que algunos están convencidos de la existencia de un fuerte corporativismo médico.

El modelo descrito en este artículo tiene varias similitudes con el Modelo de Creencias de Salud (Becker, Maiman 1975), y ha sido destacado por otros autores (Salmon et al. 1999), pero los somatizadores presentar rasgos específicos. El efecto de la transmisión de información del médico es además notable. Los sentimientos negativos que estos pacientes producen en los profesionales de la salud (García-Campayo 1998) influencia a los somatizadores para incrementar sus demandas, las cuales vuelven a producir más sentimientos negativos en los médicos, y el proceso continúa indefinidamente. Así que es necesario conocer el efecto de las interacciones médicas en los diferentes tipos de somatizadores (Salmon et al. 1999).

La principal limitación de este estudio puede ser su validez, una cuestión irresoluble en los estudios cualitativos. Sin embar- 
go, para minimizar este problema hemos seguido los siguientes criterios:

- Triangulación: Diferentes métodos de recogida de datos (en este caso entrevistas en profundidad y grupos focales) se usaron para compensar la debilidad de un método con otro y viceversa. A pesar de las críticas de la triangulación como una prueba pura de validación, se acepta porque asegura la compresión del tema y permite un análisis más profundo de los datos.

- Validación respondente: El análisis de los datos obtenidos durante las entrevistas en profundidad se ofreció a los participantes de los grupos focales y la reacción de los participantes del análisis se incorporó en el estudio.

- Análisis de casos desviados: Una de las tácticas más importantes para mejorar la calidad de las explicaciones de los estudios cualitativos es buscar y discutir los elementos de los datos que parecen contradecir las explicaciones emergentes del fenómeno a estudio. El análisis de los casos desviados modifica las conclusiones generales obtenidas del resto de los pacientes.

La conclusión principal es que hay varios patrones bien definidos que explican por qué algunos pacientes solicitan más pruebas complementarias a su médico general o la derivación a otros especialistas. Estos patrones modulan las expectativas de los pacientes sobre sus médicos. El conocimiento de estos patrones puede permitir un mejor tratamiento de estas personas. Sin embargo, hay un importante número de pacientes que no presentan patrones de comportamiento identificables específicos y que tienen que ser tratados basándose en las experiencias personales del médico. Nuevos estudios cualitativos para confirmar las características y prevalencia de los subgrupos descritos en este artículo parecen necesarios.

\section{Bibliografía}

BARSKY, A.J., WYSHAK, G., KLERMAN, G.I. Medical and psychiatric determinants of outpatient medical utilization. Medical Care 106, 24: 548-63. 1986.

BECKER, M.H., MAIMAN, L.A. Socio-behavioural determinants of compliance with health and medical care recommendations. Medical Care, 13: 10-24, 1975.

BRIDGES, K.W., GOLDBERG, D.P. Somatic presentation of DSM-III psychiatric disorders in primary care. $J$. Psychosom. Res., 29: 563-569, 1985.

CRAIG, T.K.J., BOARDMAN, A.P., DALY-JONES, O., DRAKE, H. The South London somatization study: I. Longitudinal course and the influence of early life experience. Br. J. Psychiatry, 163, 579-88, 1993.

CROMARTY, I. What do patients think about during their consultations? A qualitative study. Br. J. Gen. Pract., 46: 525-28, 1996.

ESCOBAR, J., CANINO, G. Unexplained physical complaints. Psychopathology and epidemiological correlates. Br. J. Psychiatry, Suppl: 24-27, 1989.

GARCÍA-CAMPAYO, J., CAMPOS, R., MARCOS, G., PÉREZ-ECHEVERRÍA, M.J., LOBO, A., GMPPZ. Somatisation in primary care in Spain. II. Differences between somatisers and psychologisers. Br. J. Psychiatry, 168: 348$353,1996$.

GARCÍA-CAMPAYO, J., LARRUBIA, J., LOBO, A., PÉREZ-ECHEVERRÍA, M.J., CAMPOS, R., GMPPZ. Attribution in somatisers: stability and relationship to outcome at 1-year follow-up. Acta Psychiatr. Scand., 95: 433438, 1997.

GARCÍA-CAMPAYO, J., SANZ CARRILLO, C., YOLDI, A., LÓPEZ-AYLÓN, R., MONTÓN, C. Management of somatisers in primary care: are family doctors motivated? Aust. N. Zeal. J. Psychiatry, 32: 528-533, 1998.

GARCÍA-CAMPAYO, J., SANZ CARRILLO, C. A review of the difference between somatizing and psychologizing patients in primary care. Int'1 J. Psychiatr. Med., 29: 337-45, 1993.

GARCÍA-CAMPAYO, J., SANZ CARRILLO, C. The use of alternative medicines by somatoform disorder patients in Spain. Br. J. Gen. Pract., 50: 487-88, 2000.

LIPOWSKI, Z.J. Somatization: the concept and its clinical application. Am. J. Psychiatry, 145: 1358-68, 1988.

LOBO, A., CAMPOS, R., PÉREZ-ECHEVERRÍA, M.J., IZUZQUIZA, J., GARCÍA-CAMPAYO, J., SAZ, P., MARCOS, G. A new interview for the multiaxial assess- 
ment of psychiatric morbidity in medical settings. Psychol. Med., 23: 505-510, 1993.

LOBO, A., GARCÍA-CAMPAYO, J., CAMPOS, R., MARCO, G., PÉREZ-ECHEVERRÍA, M.J., GMPPZ. Somatization in primary care in Spain. I: Estimates of prevalence and clinical characteristics. Br. J. Psychiatry, 168: 344-348, 1996.

MAYOU, R., BASS, C., SHARPE, M. Overview of epidemiology, classification and etiology. In: Treatment of functional somatic symptom. Eds. R. Mayou, C. Bass, M. Sharpe. Oxford: Oxford University Press, 1995.

MAYS, N., POPE, C. (eds.). Qualitative research in health care. London: British Medical Journal, 1996.

MAYS, N., POPE, C. Assessing quality in qualitative research. BMJ, 320: 50-52, 2000.

MAYS, N., POPE, C. Rigour in qualitative research. BMJ, 311: 109-12, 1995.

NEAL, R.D., HEYWOD, P.L., MORLEY, S. 'I always seem to be there' -a qualitative study of frequent attenders. Br. J. Gen. Pract., 50: 716-23, 2000.
RILEY, J. Getting, the most from your data: a handbook of practical ideas on how to analyze qualitative data. Bristol: Technical \& Educational Services, 1990.

ROBBINS, J.M., KIRMAYER, L.J. Attribution of common somatic symptoms. Psychol. Med., 21: 1029-1045, 1991.

SALMON, P., PETERS, S., STANLEY, I. Patient's perception of medical explanations for somatization disorders: qualitative analysis. BMJ, 318: 372-376, 1999.

WEBER, R.P. Basic content analysis. $2^{\text {nd }}$. Ed. Newbury Park, California: Sage, 1990.

Dirección para correspondencia:

Javier García-Campayo

E-mail: jgarcamp@arrakis.es

Avda. Pablo Ruiz Picasso 5, $7^{\circ} \mathrm{D}$

50015 Zaragoza

50009 Zaragoza

ESPAÑA 